\title{
Estrategias de cambio en la cultura jurídica: imediación voluntaria u obligatoria? *
}

M. I. BERGOGLIO, N. BARMAT, J. CARBALLO, M. SÁNCHEZ Y J. L. VILANOVA**

\section{RESUMEN}

La finalidad de este artículo es discutir los efectos de la concurrencia voluntaria u obligatoria al proceso de mediación. La investigación fue realizada en la provincia argentina de Córdoba, donde la ley № $\mathbf{8 8 5 8}$ establece como regla la mediación como instancia voluntaria, previendo en tres circunstancias específicas la obligatoriedad de la misma.

Empleando datos brindados por el Centro Judicial de Mediación, se analizaron 568 causas procesadas en esa institución entre 1998 y diciembre 2001. La información disponible permitió analizar el impacto de la obligatoriedad sobre los resultados y duración del proceso.

Igualmente, se efectuó una encuesta a partes con experiencia en mediación (206 casos). Esta información se emplea para discutir el efecto de la voluntariedad sobre la evaluación que hacen los actores del proceso en el que les tocó intervenir. El análisis se completa con la revisión de las opiniones de abogados y mediadores (204 casos), sobre la concurrencia obligatoria al proceso de mediación.

\section{La cuestión de la obligatoriedad}

Los precursores de la mediación en Latinoamérica debatieron la conveniencia de insertar este sistema de resolución de conflictos - en la órbita de los poderes ejecutivos o judiciales - con carácter obligatorio o voluntario. Se reconocía tácitamente que los medios empleados en la resolución de disputas tienen fuertes vínculos con los valores culturales ${ }^{1}$, y que institucionalizar el uso de medios alternati-

\footnotetext{
* El presente artículo se publica gracias a la autorización de sus autores.

** Centro de Investigaciones Jurídicas y Sociales, Facultad de Derecho y Ciencias Sociales, Universidad Nacional de Córdoba (Argentina). La correspondencia relativa a este artículo puede dirigirse a mibergoglio@arnet.com.ar

1 Un análisis profundo de las conexiones entre los modos dominantes de resolución de conflictos y los valores culturales puede encontrarse en Garapon (1997).
} 
vos requería no sólo modificaciones normativas, sino, más profundamente, un cambio cultural. Se trataba entonces de arbitrar los medios más idóneos para alentar un cambio en la cultura adversarial predominante en nuestras sociedades, conjurando los peligros derivados del «transplante» de instituciones.

Esta cuestión no resulta menor en una región donde la importación de normas sin consideración de su adecuación a la cultura legal local tiene una larga tradición, no desconectada del bajo aprecio por la legalidad y el Estado de derecho ${ }^{2}$. La cuestión permanece abierta al debate y las soluciones adoptadas difieren en cada Estado de la región; existiendo, a su vez, un número considerable de países que aún no han consagrado una normativa específica en uno u otro sentido (López González, Cholakian et al, 2002).

En Argentina, el régimen federal vigente permite la coexistencia de distintos ordenamientos procesales en el ámbito nacional y en las provincias. De esta manera, existen diversos regímenes para la instancia de mediación. Mientras la ley nacional 24573 la instituye con carácter obligatorio como instancia prejurisdiccional, las provincias de Chaco y Santa Fe asignaron carácter totalmente voluntario a la mediación como sistema para la resolución alternativa de conflictos.

En Córdoba, la cuestión suscitó amplios debates en el momento del tratamiento parlamentario de la ley de mediación. Los partidarios de la obligatoriedad argüían la necesidad de imponer el régimen para contribuir a la descongestión de los tribunales; otras posiciones destacaban el carácter esencialmente voluntario de los mecanismos de resolución alternativa de conflictos. Finalmente, la ley provincial 8858 creó un régimen mixto, donde tanto el poder Ejecutivo como el Judicial - este último a través del Centro Judicial de Mediación (CJM) tienen atribuciones para procesar conflictos. Centrándonos en el ámbito judicial, se prevé que el sometimiento de causas a la mediación tiene en principio carácter voluntario para las partes. Pero también se establece que será obligatorio concurrir a la instancia en tres supuestos: juicios de monto reducido, causas con beneficio de litigar sin gastos y cuando el juez competente lo decida ${ }^{3}$.

Como puede verse, las diferencias de criterios en materia de política procesal aún subsisten; tanto quienes bregan por la obligatoriedad como quienes sostienen el criterio de voluntariedad absoluta, cuentan con argumentaciones sólidas en uno u otro sentido. No obstante, el debate excede el ámbito de lo procesal, en tanto implica una discusión acerca de la mejor estrategia para iniciar un proceso de cambio en la cultura jurídica.

Seleccionar la obligatoriedad de la instancia supone confiar en la capacidad de las normas escritas para provocar transformaciones en la mentalidad dominante. Quienes así optan creen en la posibilidad de inducir cambios en los valores y normas sociales respecto a lo jurídico por decisión parlamentaria. Esta concepción - que ha sido la dominante por mucho tiempo entre los juristas latinoamericanos - sobrevalora la capacidad del Estado para moldear a la sociedad, y no toma en cuenta los riesgos de los trasplantes normativos.

En cambio, quienes prefieren el régimen voluntario reconocen los límites que costumbres y valores en torno al derecho ponen muchas veces a los proyectos legislativos de cambio. Aceptan que las normas escritas pueden cumplir una función educati-

2 Para una discusión del impacto de los trasplantes jurídicos sobre la cultura legal, ver Bergoglio (2003b), cap. 3. El detallado estudio de Berkovitz, Pistor y Richard (2002) presenta evidencia empírica sobre los efectos de los trasplantes jurídicos en 49 sociedades diferentes, entre las que se encuentran nueve países latinoamericanos: Argentina, Brasil, Chile, Colombia, Ecuador, México, Perú, Uruguay y Venezuela.

3 El análisis detallado del régimen legal de la mediación en Córdoba puede consultarse en Barmat y Rivero (2002). 
va, planteando nuevas formas de organización o modelos valorativamente deseables. No obstante, son conscientes de que como en todos los procesos educativos, reconocer la libertad de las personas conduce a resultados más sólidos y aspiran a que estos nuevos modos de resolución de conflictos desarrollados en otras sociedades sean genuinamente aceptados por sus usuarios. Seleccionan entonces una estrategia de cambio cultural a mediano plazo, mucho más lenta, aunque probablemente exenta de los efectos negativos asociados al trasplante brusco de instituciones.

El presente artículo pretende aportar a este debate, señalando algunas consecuencias que la opción entre voluntariedad y obligatoriedad tiene sobre la dinámica procesal de la mediación. Con ese objeto, se describen los efectos de la obligatoriedad sobre la duración y los resultados de la mediación, utilizando datos sobre las causas procesadas desde el año 2001, extraídos de la base de datos con que se gestiona en el CJM el movimiento de expedientes. También se emplearon las estadísticas proporcionadas por el CJM para los casos tramitados durante la experiencia piloto.
Esta información posibilitó la construcción de una muestra aleatoria de partes intervinientes en las causas sometidas a mediación judicial, antes y después de la sanción de la ley 8858. El trabajo de campo, realizado en 2001, permitió efectuar 206 entrevistas con las partes. Asimismo, se realizó una encuesta a operadores legales: 154 abogados y 48 mediadores. Estos materiales permiten recoger la perspectiva de los usuarios, destinatarios finales de las innovaciones en la administración de justicia, sobre la cuestión de la obligatoriedad.

\section{Diferencias entre causas obligatorias y voluntarias}

La implementación inicial de la mediación, realizada en Córdoba desde el año 1998 tuvo en la provincia carácter enteramente voluntario. Con la vigencia de la ley 8858 - que estableció la obligatoriedad de la instancia para ciertas causas - el flujo de causas creció significativamente. La institucionalización de la experiencia introdujo además otros cambios, pues la tarea de los mediadores, que inicialmente era gratuita, pasó a ser remunerada.

Total de causas en mediación judicial

\begin{tabular}{|l|c|c|c|}
\hline Año & Voluntarias* & Obligatorias & Total \\
\hline Experiencia piloto, 1998-2000 & 130 & -- & 130 \\
\hline 2001 & 93 & 345 & 438 \\
\hline 2002 & 350 & 1109 & 1459 \\
\hline 2003 (ene-jun) & 166 & 891 & 1057 \\
\hline Total desde la ley 8858 & 609 & 2345 & 2954 \\
& $20.6 \%$ & $79.3 \%$ & $100 \%$ \\
\hline Total general & 739 & 2345 & 3084 \\
& $23.9 \%$ & $76.1 \%$ & $100 \%$ \\
\hline Casos radicados en el Centro de Mediación Judicial Córdoba Capital \\
*Incluye casos de familia derivados en función de la ley 9032. \\
\hline
\end{tabular}


Actualmente el grueso del trabajo del Centro se concentra en los casos derivados de manera obligatoria. La cantidad de casos donde las partes eligen la mediación judicial de manera completamente voluntaria es relativamente baja; apenas al $8.4 \%$. Las prescripciones de la ley 9032 , que permiten a las partes concurrir al CJM para cumplimentar allí la instancia de conciliación prejurisdiccional en casos del fuero de familia si así lo desean, han llevado el número de causas donde existe algún grado de voluntariedad al $21 \%{ }^{4}$.

El análisis que se efectúa a continuación se concentra en las 568 causas procesadas en el CJM entre 1998 y diciembre 2001, es decir, antes y después de la vigencia de la ley 8858. Este conjunto incluye un $38 \%$ de casos derivados al Centro de manera voluntaria, la mayoría de ellos proveniente del campo civil. Se incluyen aquí procesos que cubren una gran variedad de materias, como desalojos, ejecutivos, declaratorias de herederos o nulidades.

La mayoría de los conflictos atendidos en el CJM tienen una clara naturaleza patrimonial: la proporción de causas en las que el reclamo tiene un monto indeterminado no llega al $30 \%$. El análisis de los montos según forma de derivación permite observar que las causas donde las partes concurren voluntariamente a esta instancia tienen en promedio montos algo más bajos. La proporción de disputas donde las partes no especifican un monto determinado para sus reclamos resulta también algo mayor en este grupo. Ello sugiere que el uso voluntario de este instituto resulta más probable en cuestiones de menor significación económica.

\section{Comparación de los montos involucrados}

Monto
\begin{tabular}{|l|r|r|r|c|}
\hline Forma de derivación & \multicolumn{1}{|c|}{$\mathrm{N}^{*}$} & \multicolumn{1}{c|}{ Mínimo } & Máximo & Media \\
\hline Voluntaria & 51 & 200.00 & 361000.0 & 67691.36 \\
\hline Obligatoria & 257 & 62.57 & 2737183 & 82732.74 \\
\hline Total & 308 & 62.57 & 2737183 & 80242.12 \\
\hline
\end{tabular}

Causas 1-438, año 2001 -

${ }^{*}$ ) Sólo se incluyen las causas de monto determinado

El análisis de la duración de las causas diferenciadas según su forma de derivación es útil para observar algunos efectos de la obligatoriedad. Las causas llegadas voluntariamente al CJM tienen una duración media de 58 días, e implican la realiza- ción de un promedio de tres audiencias. En cambio las causas obligatorias se extienden durante 51 días, durante los cuales se efectúa un promedio de 2.3 audiencias. La diferencia entre ambos tipos de causas es estadísticamente significativa ${ }^{5}$.

Duración de las causas según forma de derivación

Días entre el ingreso y el egreso

\begin{tabular}{|l|r|r|r|r|}
\hline Forma de derivación & \multicolumn{1}{|c|}{$\mathrm{N}$} & \multicolumn{1}{c|}{ Mínimo } & \multicolumn{1}{c|}{ Máximo } & \multicolumn{1}{c|}{ Media } \\
\hline Voluntaria & 182 & 9 & 207 & 57.93 \\
\hline Obligatoria & 253 & 10 & 247 & 50.66 \\
\hline Total & 439 & 9 & 247 & 53.79 \\
\hline
\end{tabular}

Causas 1998 - 2001 - Incluye sólo causas terminadas en abril 2002.

4 Debe observarse, sin embargo, que la concurrencia al CJM en los procesos de familia aún no judicializados habilitada por la ley 9032 no supone prácticamente la elección por las partes de una forma alternativa para resolver su conflicto. Más bien, la opción por llevar adelante en el CJM esta etapa prejurisdiccional representa la elección de un trámite más rápido de su problema, concurriendo a una oficina de la administración de Justicia percibida como más rápida y eficiente.

5 Para mayores detalles sobre la forma en que se midió la duración de las causas, ver Bergoglio 2003a. La correlación entre la duración del proceso y la forma de derivación es de .115, significativa para $\mathrm{p}<.016$. 
Resultados según forma de derivación

\begin{tabular}{|l|c|c|c|}
\hline & Voluntarias & Obligatorias & Total causas* \\
\hline Causas con acuerdo & $43.6 \%$ & $29.7 \%$ & $35.4 \%$ \\
\hline Causas sin acuerdo & $42.1 \%$ & $42.1 \%$ & $42.2 \%$ \\
\hline No comparecencia & $10.9 \%$ & $12.8 \%$ & $12.3 \%$ \\
\hline Desistimiento & $2.0 \%$ & $7.5 \%$ & $5.1 \%$ \\
\hline No mediables & $1.5 \%$ & $7.9 \%$ & $5.1 \%$ \\
\hline Total & $100.0 \%$ & $100.0 \%$ & $100.0 \%$ \\
\hline $\begin{array}{l}(*) \mathrm{N}=468 \text { causas tramitadas en el CJM, } 1998-2001 . \text { Incluye sólo causas } \\
\text { terminadas. } \\
\text { Chi- cuadrado significativo para } \mathrm{p}<.0000 .\end{array}$ \\
\hline
\end{tabular}

Estos datos indican que cuando las partes concurren voluntariamente a la mediación, su compromiso con la búsqueda de alternativas es mayor; en consecuencia, se registra mayor número de audiencias y el proceso se alarga. En cambio, los procesos donde la ley dispone de manera obligatoria la instancia, resultan algo más cortos, reflejando las situaciones en que alguna de las partes, disconforme con este método de resolución de conflictos, se limita a concurrir a la audiencia inicial, desistiendo de la instancia.

Generalmente, se invoca en defensa de la mediación su carácter no adversarial. También se alude a la posibilidad de evitar el juicio, lo que redunda en un acortamiento global del trámite. Ambos resultados favorables se obtienen cuando se llega a un acuerdo entre las partes. Como puede verse en el cuadro adjunto, la proporción de acuerdos es claramente superior entre quienes concurren voluntariamente a la mediación ${ }^{6}$. En estas causas, resulta además baja la proporción de casos en los que alguna de las partes no concurrió a la instancia.

La tasa de acuerdos baja al 30\% entre las causas obligatorias. La incomparecencia y el desistimiento, actitudes que indican la falta de interés en la instancia, alcanzan igualmente valores significativos. De hecho, $28 \%$ de los casos derivados obligatoriamente al CJM terminan sin que las partes se hayan sentado finalmente alrededor de la misma mesa de la negociación. Estos datos ilustran la incidencia que la forma de derivación tiene sobre los resultados del proceso. La relación entre ambas variables es clara y alcanza significación estadística?

\footnotetext{
6 Del mismo modo, en la provincia de Santa Fe, donde las causas ingresadas a solicitud de parte constituyen el $95 \%$, la tasa de acuerdos llega al $53 \%$.

7 Chi cuadrado $=29.341$, significativo para $\mathrm{p}<.0000$. Correlación: Gamma $=.278$, nivel de significación .000 .
} 
Obligatoriedad y resultados en la mediación

\begin{tabular}{|l|c|c|c|}
\hline & $\begin{array}{c}\text { Voluntarias, } \\
\text { Córdoba }\end{array}$ & $\begin{array}{c}\text { Obligatorias, } \\
\text { Córdoba }\end{array}$ & $\begin{array}{c}\text { Obligatorias* } \\
\text { Ciudad Bs. As. }\end{array}$ \\
\hline Causas con acuerdo & $43.6 \%$ & $29.7 \%$ & $27.27 \%$ \\
\hline Causas sin acuerdo & $42.1 \%$ & $42.1 \%$ & $41.31 \%$ \\
\hline Causas no mediadas & $14.3 \%$ & $28.2 \%$ & $31.42 \%$ \\
\hline Total & $100.0 \%$ & $100.0 \%$ & $100.0 \%$ \\
\hline $\begin{array}{l}\text { (*) Causas derivadas obligatoriamente a mediación en los fueros civil y } \\
\text { comercial de la ciudad de Buenos Aires, (Mayo 1996 - Diciembre 2000). } \\
\text { Datos informados por el Ministerio de Justicia. }\end{array}$ \\
\hline
\end{tabular}

Es interesante observar la semejanza de estas cifras con las registradas en la ciudad de Buenos Aires. En las dos jurisdicciones, las causas derivadas obligatoriamente a mediación obtienen una proporción de arreglos negociados entre las partes inferior al $30 \%$.

En síntesis, puede decirse que los casos llegados voluntariamente a la mediación suelen tener menor significación económica, sea porque se trata de conflictos donde las partes no cifran en dinero sus pretensiones, o de disputas por montos relativamente bajos. La concurrencia voluntaria se traduce en un mayor compromiso con el logro de soluciones, y, en consecuencia, el proceso es más largo, pues demanda mayor número de audiencias. En estas condiciones, las probabilidades de obtener un arreglo consensuado entre las partes son también mayores.

\section{Mediados obligados y voluntarios}

La evaluación que las partes efectúan sobre su experiencia en estos foros informales puede ser variada. En la mayoría de los casos se destaca el ahorro de tiempo y costos, el carácter no adversarial de estos procesos, así como las oportunidades de participación que proveen a las partes (Menkel-
Meadow, 2000). Igualmente se remarca su capacidad para producir soluciones creativas y flexibles, que preserven los vínculos sociales existentes.

La valoración positiva no es sin embargo general. Así, Engle Merry (1990), en su clásico estudio acerca de las experiencias de los trabajadores en mediación en Nueva Inglaterra, mostró que la presión excesiva hacia el tratamiento informal de los conflictos puede acentuar las vivencias de exclusión del sistema judicial. La ley 8858, que vuelve obligatoria la mediación en las causas que interesan a litigantes de bajos recursos, hace que este riesgo resulte significativo en el caso cordobés. Por ese motivo, este apartado se destina a revisar las diferencias entre concurrentes obligados y voluntarios, tanto en general como en su evaluación de la experiencia.

Quienes han concurrido al CJM se concentran en ciertos grupos sociales. Predominan los hombres $(66 \%)$; aunque en las causas voluntarias la distribución por géneros resulta algo más equilibrada. Este dato no sorprende, ya que en Córdoba, las concepciones tradicionales de las relaciones de género marcan diferencias en el contacto con tribunales (Bergoglio, 1999). El 60\% de los entrevistados reconoce niveles de ingreso familiares superiores a 
los demarcatorios de la línea de pobreza para una familia de 4 personas ${ }^{8}$. También este dato es esperable, en tanto los grupos de mayores ingresos son usuarios más intensos del servicio de justicia (Bergoglio, 1997).

Dominan las personas maduras, con más de 40 años. Se trata de una población con un nivel educativo relativamente alto, cuya participación en la vida económica es significativa, siendo el porcentaje de desocupados y económicamente no activos menor del que se registra en la población general.

Si se revisa el perfil de quienes han concurrido voluntariamente a la mediación, se verá que sus niveles educativos y de ingresos resultan algo más altos que el de los usuarios obligados, revelando que la aceptación de esta institución resulta más rápida en los sectores sociales medios y altos.

El conocimiento de los propios derechos es un elemento importante en el proceso de movilización del Derecho. Para poder demandar justicia, es necesaria una construcción de la identidad personal como ciudadano. Según han mostrado los estudios sobre socialización legal (Kourilsky, 1997) la confianza en los derechos es una variable fuertemente influida por la educación. Entre quienes han concurrido al CJM, la difusión de la educación es amplia y el conocimiento de los propios derechos tiene un nivel bastante significativo. Esta proporción es aún mayor entre quienes han concurrido voluntariamente a la mediación.

Características generales de las partes

\begin{tabular}{|c|c|c|c|c|}
\hline & \multicolumn{2}{|c|}{ Forma de derivación } & \multirow[b]{2}{*}{ Total } \\
\hline & & Voluntaria & Obligatoria & \\
\hline \multirow{2}{*}{ Sexo } & Hombre & $58.7 \%$ & $72.8 \%$ & $66.5 \%$ \\
\hline & Mujer & $41.3 \%$ & $27.2 \%$ & $33.5 \%$ \\
\hline \multicolumn{2}{|l|}{ Total } & $100.0 \%$ & $100.0 \%$ & $100.0 \%$ \\
\hline \multirow{3}{*}{$\begin{array}{l}\text { Edad } \\
\text { entrevistado }\end{array}$} & Hasta 40 años & $28.3 \%$ & $29.5 \%$ & $28.9 \%$ \\
\hline & 40 a 55 años & $43.5 \%$ & $43.8 \%$ & $43.6 \%$ \\
\hline & Más de 55 años & $28.3 \%$ & $26.8 \%$ & $27.5 \%$ \\
\hline \multicolumn{2}{|l|}{ Total } & $100.0 \%$ & $100.0 \%$ & $100.0 \%$ \\
\hline \multirow{4}{*}{$\begin{array}{l}\text { Educación } \\
\text { del } \\
\text { entrevistado }\end{array}$} & Universitaria completa & $42.4 \%$ & $23.7 \%$ & $32.0 \%$ \\
\hline & Superior & $23.9 \%$ & $32.5 \%$ & $28.6 \%$ \\
\hline & Secundaria & $20.7 \%$ & $29.8 \%$ & $25.7 \%$ \\
\hline & Primaria & $13.0 \%$ & $14.0 \%$ & $13.6 \%$ \\
\hline \multicolumn{2}{|l|}{ Total } & $100.0 \%$ & $100.0 \%$ & $100.0 \%$ \\
\hline \multirow{5}{*}{$\begin{array}{l}\text { Nivel de } \\
\text { ingresos de } \\
\text { la familia }\end{array}$} & Más de 2500 & $10.9 \%$ & $8.8 \%$ & $9.7 \%$ \\
\hline & $1500-2500$ & $15.2 \%$ & $14.0 \%$ & $14.6 \%$ \\
\hline & $500-1500$ & $39.1 \%$ & $43.9 \%$ & $41.7 \%$ \\
\hline & Hasta 500 & $27.2 \%$ & $22.8 \%$ & $24.8 \%$ \\
\hline & $\mathrm{Ns} / \mathrm{nc}$ & $7.6 \%$ & $10.5 \%$ & $9.2 \%$ \\
\hline \multicolumn{2}{|l|}{ Total } & $100.0 \%$ & $100.0 \%$ & $100.0 \%$ \\
\hline
\end{tabular}

$\mathrm{N}=206$ partes

$8 \quad$ En el momento de recolección de datos, el valor de la línea de pobreza para una familia de cuatro miembros residente en la Capital Federal era de 598 pesos (Clarín, 10 de mayo de 2002). Las familias que percibían ingresos por debajo de ese límite pueden considerarse pobres. 
Quienes han tenido mayor experiencia de contacto con el mundo tribunalicio tienen igualmente una actitud más activa en la defensa de sus derechos. Al igual que el conocimiento de los propios derechos, la familiaridad con el sistema jurídico ${ }^{9}$ aumenta con la educación y es más frecuente entre quienes concurrieron voluntariamente a la mediación.
En resumen, puede decirse que la concurrencia voluntaria a la mediación es más frecuente entre las mujeres - que aprecian especialmente el estilo no adversarial - $y$ entre los grupos de mayor nivel socioeconómico, así como entre quienes tienen mayor familiaridad con el mundo jurídico.

Familiaridad con el sistema jurídico según forma de derivación

\begin{tabular}{|c|c|c|c|c|}
\hline & \multicolumn{2}{|c|}{ Forma de derivación } & \multirow[t]{2}{*}{ Total } \\
\hline & & Voluntaria & Obligatoria & \\
\hline \multirow{3}{*}{$\begin{array}{l}\text { Conoce sus } \\
\text { Derechos } \\
\text { Total }\end{array}$} & Muy bien o bien & $\% \quad 56.5$ & $\% 47.4$ & $\% \quad 51.5$ \\
\hline & Regular & 32.6 & 34.2 & 33.5 \\
\hline & Poco o nada & 10.9 & 18.4 & 15.0 \\
\hline & & 100.0 & 100.0 & 100.0 \\
\hline \multirow{4}{*}{$\begin{array}{l}\text { Familiaridad } \\
\text { con el } \\
\text { sistema } \\
\text { jurídico }\end{array}$} & Nula & 7.6 & 13.2 & 10.7 \\
\hline & Baja & 29.3 & 43.9 & 37.4 \\
\hline & Media & 47.8 & 30.7 & 38.3 \\
\hline & Alta & 15.2 & 12.3 & 13.6 \\
\hline \multicolumn{2}{|l|}{ Total } & 100.0 & 100.0 & 100.0 \\
\hline
\end{tabular}

$\mathrm{N}=206$ partes

\section{La evaluación de la mediación}

El proceso de la mediación supone una instancia en la que se tramitan conflictos, a veces muy agudos, y en la que legos y letrados participan buscando - o no - un arreglo negociado. Constituye entonces un evento en el que las dimensiones a evaluar son múltiples. El cuestionario permitió explorar la opinión de las partes en torno al funcionamiento del CJM, así como el papel llevado adelante por los mediadores. Aquí se resumen esas evaluaciones, comparando las opiniones de mediados obligados y voluntarios.

El hecho de que una causa judicial se derive a mediación supone que el Estado comparte su po- testad antes exclusiva en la resolución de conflictos con otros operadores legales privados. No obstante, en la mediación judicial, el trámite de los procesos continúa haciéndose en el espacio físico de los tribunales, y, por ello mismo, encuadrado dentro de las formas organizacionales que la administración de justicia ofrece y bajo la supervisión de sus funcionarios.

La evaluación de la experiencia en el CJM por parte de los mediados puede considerarse muy positiva, ya que tres cuartas partes de los entrevistados se declaran completamente satisfechos con las diferentes dimensiones incluidas. La forma de trato del personal, el ambiente de los encuentros y la infor-

$9 \quad$ La familiaridad con el sistema jurídico se midió con un índice que combina las variables conocimiento de los propios derechos, conocimiento de la existencia de ayuda legal gratuita y de la mediación. Varía desde 0 (nula familiaridad) a 3 (alta familiaridad), con una media de 1.54. 
Evaluación de la experiencia en el CJM

\begin{tabular}{|l|c|c|c|}
\hline \multirow{2}{*}{ Opinión sobre } & \multicolumn{2}{|c|}{ Forma de derivación } & \multirow{2}{*}{ Total } \\
\cline { 2 - 3 } & Voluntaria & Obligatoria & \\
\hline La forma en que el personal lo trató & $84.8 \%$ & $82.5 \%$ & $83.5 \%$ \\
\hline El ambiente de los encuentros & $82.6 \%$ & $78.9 \%$ & $80.6 \%$ \\
\hline La flexibilidad de horarios de las audiencias & $83.7 \%$ & $74.6 \%$ & $78.6 \%$ \\
\hline La información de la mediación y la situación & $73.9 \%$ & $74.6 \%$ & $74.3 \%$ \\
\hline $\mathrm{N}=206$ encuestados & & \\
\hline
\end{tabular}

mación brindada han resultado satisfactorios para una amplia mayoría de los encuestados.

Como se observa en el cuadro adjunto, tanto mediados voluntarios como obligatorios coinciden en sus apreciaciones favorables sobre la actividad del CJM, siendo apenas más altos los niveles de satisfacción entre quienes se acercaron voluntariamente. Las diferencias entre ambos grupos son escasas y no alcanzan significación estadística.

El rol que los mediadores cumplen durante la mediación resulta central y son muchas las expectativas que pesan sobre ellos. Se les pide imparcialidad, tacto, paciencia, creatividad en la búsqueda de soluciones, respeto de la legislación pertinente, de la voluntad de las partes y también de sus demandas de privacidad. Se trata, pues, de un papel complejo, aunque de baja tradición en el país.

Como se expuso anteriormente, la mayoría de las dimensiones evaluadas en el desempeño del mediador obtienen respuestas favorables cercanas al $70 \%$, aunque en general, la imagen de los mediadores resulta algo menos positiva que la obtenida por el funcionamiento del CJM. Respeto de la confidencialidad y paciencia en las negociaciones, son los rubros mejor apreciados. La alta valoración que recibe el trato igualitario en el contexto de la mediación sugiere que éste es menos frecuente de lo que cabría esperar en un estado democrático.

Evaluación de la actuación del mediador

\begin{tabular}{|l|c|c|c|}
\hline \multirow{2}{*}{ Opinión sobre } & Forma de derivación & \multirow{2}{*}{ Total } \\
\cline { 2 - 3 } & Voluntaria & Obligatoria & \\
\hline Su respeto de la confidencialidad & $80.2 \%$ & $72.6 \%$ & $76.0 \%$ \\
\hline La paciencia demostrada durante las negociaciones & $83.5 \%$ & $68.1 \%$ & $75.0 \%$ \\
\hline La forma en que trataban a ambas partes por igual & $76.9 \%$ & $66.4 \%$ & $71.1 \%$ \\
\hline El tiempo destinado a las negociaciones & $76.9 \%$ & $65.5 \%$ & $70.6 \%$ \\
\hline Su habilidad para manejar pacíficamente la situación & $73.6 \%$ & $66.4 \%$ & $69.6 \%$ \\
\hline La forma en que plantearon el tema de sus honorarios & $59.7 \%$ & $53.6 \%$ & $56.0 \%$ \\
\hline Las opciones de solución surgidas & $56.0 \%$ & $45.1 \%$ & $50.0 \%$ \\
\hline N = 206 encuestados & & & \\
\hline Porcentajes de entrevistados que se declararon completamente satisfechos & & \\
\hline
\end{tabular}


En cambio, la forma en que los mediadores plantearon el tema de sus honorarios y el manejo de las soluciones posibles surgidas reciben puntuaciones más bajas: la mitad de los entrevistados se declara satisfecho con estos aspectos.

El cuadro anexo muestra igualmente que mediados obligados y voluntarios difieren en su apreciación del rol cumplido por el mediador. La reducción de evaluaciones altamente positivas en el primer grupo es visible, y sugiere que la satisfacción con la experiencia se reduce entre quienes concurren obligatoriamente.

Tras haber transitado por estos foros alternativos, las partes construyen una imagen de la mediación como mecanismo de resolución de disputas, relativamente independiente de su evaluación de la actuación de los operadores legales participantes en el caso.

Como puede verse en el cuadro adjunto, los entrevistados valoran la presencia en su proceso de mediación de atributos considerados centrales para su legitimación: su carácter equitativo, el grado de participación que permitieron a los interesados, así como la celeridad de la respuesta procedimental del Estado. Todas estas dimensiones recibieron menciones positivas superiores al $60 \%$, tanto entre quienes concurren voluntariamente como de manera obligatoria.

La mirada sobre los costos resulta más crítica, al igual que ocurriera en lo relativo a los honorarios de los mediadores, que reciben porcentajes de opiniones positivas cercanos al $40 \%$. Las quejas frente a estos aspectos son especialmente notables entre quienes han concurrido obligatoriamente a esta instancia, quienes perciben que los costos y tiempos que la mediación insume se sobreagregan a los generados por el proceso judicial.

En resumen, los datos de encuesta permitieron observar que quienes han transitado por una experiencia de mediación tienen una perspectiva positiva sobre este proceso. La evaluación del rol cumplido por mediadores y funcionarios del CJM, así como sobre las características generales del proceso, resulta mayoritariamente favorable. Las diferencias en las evaluaciones que efectúan mediados

Evaluación de la mediación como proceso

\begin{tabular}{|c|c|c|c|}
\hline \multirow{2}{*}{ Opinión sobre } & \multicolumn{2}{|c|}{ Forma de derivación } & \multirow{2}{*}{ Total } \\
\hline & Voluntaria & Obligatoria & \\
\hline La equidad del proceso de mediación & $67.4 \%$ & $64.9 \%$ & $\begin{array}{c}66.0 \\
\%\end{array}$ \\
\hline La forma en que usted pudo participar en la solución del problema & $65.2 \%$ & $66.7 \%$ & $\begin{array}{c}66.0 \\
\%\end{array}$ \\
\hline El tiempo pasado hasta que tuvieron la primera audiencia & $62.0 \%$ & $63.2 \%$ & $\begin{array}{c}62.6 \\
\%\end{array}$ \\
\hline $\begin{array}{l}\text { El tiempo que llevó la solución del asunto, una vez iniciada la } \\
\text { mediación }\end{array}$ & $56.0 \%$ & $39.6 \%$ & $\begin{array}{c}47.0 \\
\%\end{array}$ \\
\hline Los costos económicos de este modo de solución de conflictos & $52.6 \%$ & $38.9 \%$ & $\begin{array}{c}44.5 \\
\%\end{array}$ \\
\hline La variedad de opciones de solución surgidas & $44.6 \%$ & $38.6 \%$ & $\begin{array}{c}41.3 \\
\%\end{array}$ \\
\hline
\end{tabular}


Satisfacción con la experiencia en mediados obligados y voluntarios

\begin{tabular}{|c|c|c|c|c|}
\hline & & \multicolumn{2}{|c|}{ Forma de derivación } & \multirow{2}{*}{ Iotal } \\
\hline & & Voluntarla & Obligatoria & \\
\hline \multirow{3}{*}{$\begin{array}{l}\text { Satisfacción con } \\
\text { el Centro Judicial } \\
\text { de Mediación }\end{array}$} & Baja & $10.9 \%$ & $14.0 \%$ & $12.6 \%$ \\
\hline & Media & $26.1 \%$ & $24.6 \%$ & $25.2 \%$ \\
\hline & Alta & $63.0 \%$ & $61.4 \%$ & $62.1 \%$ \\
\hline \multicolumn{2}{|l|}{ Tocal } & $100.0 \%$ & $100.0 \%$ & $100.0 \%$ \\
\hline \multirow{3}{*}{$\begin{array}{l}\text { Satisfacción con } \\
\text { la acluación del } \\
\text { mediador }\end{array}$} & Baja & $17.4 \%$ & $28.9 \%$ & $23.8 \%$ \\
\hline & Media & $25.0 \%$ & $22.8 \%$ & $23.8 \%$ \\
\hline & Alta & $57.6 \%$ & $48.2 \%$ & $52.4 \%$ \\
\hline \multicolumn{2}{|l|}{ Toual } & $100.0 \%$ & $100.0 \%$ & $100.0 \%$ \\
\hline \multirow{3}{*}{$\begin{array}{l}\text { Satisfacción con } \\
\text { la actuación del } \\
\text { abogado }\end{array}$} & Bala & $34.8 \%$ & $26.3 \%$ & $30.1 \%$ \\
\hline & Media & $14.1 \%$ & $20.2 \%$ & $17.5 \%$ \\
\hline & Alta & $51.1 \%$ & $53.5 \%$ & $52.4 \%$ \\
\hline \multicolumn{2}{|l|}{ Total } & $100.0 \%$ & $100.0 \%$ & $100.0 \%$ \\
\hline \multirow{3}{*}{$\begin{array}{l}\text { Satisfacción con } \\
\text { el proceso de la } \\
\text { mediación }\end{array}$} & Baja & $30.4 \%$ & $40.4 \%$ & $35.9 \%$ \\
\hline & Media & $35.9 \%$ & $33.3 \%$ & $34.5 \%$ \\
\hline & Alta & $33.7 \%$ & $26.3 \%$ & $29.6 \%$ \\
\hline \multicolumn{2}{|l|}{ Total } & $100.0 \%$ & $100.0 \%$ & $100.0 \%$ \\
\hline
\end{tabular}

obligados y voluntarios pueden ser mejor apreciadas si se utilizan índices de satisfacción que sintetizan las respuestas para cada bloque de variables.

Para cada bloque de variables (satisfacción con la actuación del Centro, con el abogado, con el mediador, o con el proceso de la mediación) el índice resume el conjunto de respuestas "completamente satisfecho" a cada una de las preguntas detalladas más arriba. Los índices resultantes fueron ponderados tomando en cuenta el número de variables incluidas en cada uno de ellos para posibilitar su comparación.

Ambos grupos de litigantes contemplan de manera similar la tarea desempeñada por el CJM. Flexibilidad de horarios de las audiencias, ambiente de los encuentros o atención del personal son apreciadas en idéntica proporción en los dos grupos.

En cambio, mediados obligados y voluntarios difieren en su apreciación del rol cumplido por el mediador, así como en su visión global de este modo de procesar conflictos. La reducción de evaluaciones altamente satisfactorias en el primer grupo es visible, aunque no alcanza significación estadística. Estas observaciones añaden un nuevo elemento a la polémica acerca de la conveniencia de la obligatoriedad o no de la mediación.

\section{La visión de la obligatoriedad}

La cuestión acerca de la obligatoriedad que venimos discutiendo no es simplemente un debate técnico, a dilucidar mediante intervenciones de doctrinarios o expertos en política procesal. Es igualmente una discusión acerca del grado en que el poder de resolver conflictos - una de las competencias más antiguas del Estado - se comparte con otros operadores legales, distintos de los magistrados. Por ello la opinión de los ciudadanos sobre el tema resulta más significativa.

La encuesta mostró que la mayoría de las partes (56\%) estima que la mediación debería ser obligatoria de modo general. Son minoritarios en este 


\section{Visión de la obligatoriedad}

\begin{tabular}{|l|c|c|c|}
\hline \multirow{2}{*}{ ¿Cree usted que la mediación debería ser obligatoria? } & Forma de derivación & \multirow{2}{*}{ Total } \\
\cline { 2 - 3 } & Voluntaria & Obligatoria & \\
\hline Debería ser obligatorio para todos los casos, como en Bs. As. & $58.2 \%$ & $54.5 \%$ & $56.2 \%$ \\
\hline Debería ser obligatorio para algunos casos & $23.1 \%$ & $26.8 \%$ & $25.1 \%$ \\
\hline No debería ser obligatorio en ningún caso & $18.7 \%$ & $18.8 \%$ & $18.7 \%$ \\
\hline Total & 100.0 & 100.0 & 100.0 \\
\hline & & & \\
\hline
\end{tabular}

grupo los que creen que la voluntariedad debería ser la regla en todos los casos, en tanto una cuarta parte se pronuncia a favor de un sistema mixto, como el establecido por la ley 8858. Hay pocas diferencias de opinión en este tema entre quienes han concurrido al CJM de manera voluntaria o compulsiva.

Resulta claro que en las partes predomina una mirada positiva sobre este método de procesamiento de conflictos, que se pretende difundir recurriendo a la obligatoriedad de la instancia. Esta lectura se confirma por el hecho de que la opción por la obligatoriedad está asociada a la satisfacción con la experiencia, a la decisión de recomendar este método de resolución de conflictos, así como a la convicción de que amplía el acceso a la justicia ${ }^{10}$.

Los datos de la encuesta a los abogados muestran los contrastes entre la cultura legal externa y la interna. La opinión de los abogados es claramente desfavorable a la obligatoriedad generalizada de la instancia ( $45 \%)$, postura que revela su apego a las formas tradicionales de resolución de conflictos. No obstante, un tercio de los entrevistados reconoce su utilidad para ciertos tipos de disputas, como los casos de familia, cuestiones de menor cuantía o los que se plantean entre vecinos.

\section{Visión de la obligatoriedad según rol en el proceso}

\begin{tabular}{l|c|c|c|c|}
\hline $\begin{array}{l}\text { ¿Cree usted que esta forma de resolución de conflictos } \\
\text { debería ser obligatoria? }\end{array}$ & Partes & Abogados & Mediadores & Total \\
\hline $\begin{array}{l}\text { Debería ser obligatorio para todos los casos, como en Bs. } \\
\text { As. }\end{array}$ & $56.2 \%$ & $17.6 \%$ & $42.0 \%$ & $39.9 \%$ \\
\hline Debería ser obligatorio para algunos casos & $25.1 \%$ & $37.3 \%$ & $42.0 \%$ & $31.7 \%$ \\
\hline No debería ser obligatorio en ningún caso & $18.7 \%$ & $45.1 \%$ & $16.0 \%$ & $28.3 \%$ \\
\hline Total & $100.0 \%$ & $100.0 \%$ & $100.0 \%$ & $100.0 \%$ \\
\hline & $\mathrm{N}=203$ & $\mathrm{~N}=153$ & $\mathrm{~N}=50$ & $\mathrm{~N}=406$ \\
\hline
\end{tabular}

10 En los tres casos, las relaciones entre las variables presentan chi-cuadrado significativo para p<.001. Para un análisis detallado de estas evaluaciones desde la perspectiva del acceso a la justicia ver Bergoglio y Vilanova, 2004. 
La visión de los abogados revela también su clara percepción de la enorme variedad de disputas que procesa la administración de justicia, lo que desaconseja las soluciones uniformes para todos los tipos de causas. Su mayor experiencia en conflictos les permite identificar casos en que la compulsividad de la instancia redunda sólo en un alargamiento general del trámite, como cuando una de las partes carece en la práctica de posibilidades de negociar ${ }^{11}$ o en las causas civiles que dependen de la resolución de otra penal, siendo esta última no mediable. En general, esta postura dominantemente conservadora está asociada a una mirada escéptica sobre la capacidad de la mediación para ampliar el acceso a la Justicia.

Entre los mediadores, son pocos quienes se mantienen dentro del paradigma clásico de esta forma de resolución de conflictos, recomendando la voluntariedad absoluta. La mayoría de ellos admite alguna forma de obligatoriedad, que generalmente se justifica como medio de difundir una institución novedosa en nuestro medio ${ }^{12}$. De cualquier modo, su entusiasmo con la generalización de la instancia es menor del que despliegan las partes. Algunos mediadores de más experiencia señalan que la obligatoriedad disminuye la efectividad del sistema, en tanto reduce el número de acuerdos.

El contraste en la distribución de opiniones entre partes, abogados y mediadores resulta marcado, $y$ deja a la luz la ubicación de los actores frente a la distribución institucional de la potestad de resolver disputas. Los justiciables se pronuncian mayoritariamente por la obligatoriedad generalizada de la mediación, una postura que desemboca finalmente en una reducción de la esfera de asun- tos sometidos al poder de los jueces. En cambio, los abogados prefieren preservar el mayor número posible de conflictos bajo el control exclusivo de quienes, como ellos, poseen una formación jurídica. Por su parte, los mediadores - entre quienes se encuentran tanto abogados como profesionales formados en otras disciplinas - se ubican en una posición intermedia ${ }^{13}$.

Estas divergencias de opinión entre los distintos actores del proceso, cuyas demandas e intereses frente al sistema de administración de justicia son finalmente tan distintas, ilustran la complejidad de las decisiones implicadas en la regulación de la cuestión.

\section{Conclusiones}

El análisis de la experiencia cordobesa - régimen mixto que combina voluntariedad y obligatoriedad - permite señalar algunas consecuencias de una opción en uno u otro sentido. Tales consecuencias se registran tanto a nivel de las causas individualmente consideradas, como en el nivel global de la Administración de Justicia.

Si se atiende primero a las consecuencias a nivel de los casos individuales, se verá que cuando las partes concurren voluntariamente a la mediación su compromiso con la búsqueda de soluciones al conflicto es mayor. Por ello los procesos resultan algo más largos, en tanto las negociaciones insumen mayor tiempo, siendo el desistimiento o la incomparecencia menos frecuentes. En consecuencia, la proporción de casos que finalizan con un acuerdo es mayor.

11 Los casos de indemnizaciones por daños en los que intervienen compañías de seguro con sede en Buenos Aires se encuentran generalmente en esta situación, en tanto estas personas jurídicas no reconocen a los letrados que las representan en el acto capacidad suficiente para negociar de manera autónoma.

12 ...debería ser obligatoria al principio por que hay mucha resistencia por que la gente no conoce exactamente de lo que se trata, pero después de un tiempo que esto fuese así y que esté metido en la sociedad, yo creo que tiene que ser voluntario" (E46).

13 Si se analizan las opiniones frente al juicio por jurados - una cuestión que supone igualmente recortar el poder decisorio de los jueces - se encuentra una distribución muy similar entre los diversos actores del proceso, lo que refuerza la interpretación expuesta. Razones de espacio impiden presentar aquí esos datos. 
Observando ahora las consecuencias desde el nivel macro, se advertirá que cuando el legislador prefiere la voluntariedad, el perfil de los usuarios del sistema de procesamiento de disputas se modifica, al menos en las etapas iniciales de la adopción de esta innovación institucional. La decisión de llevar el propio conflicto a mediación es más probable entre las mujeres, poco afines al espíritu competitivo que el proceso tradicional implica.

Es también más probable entre los disputantes de mayor nivel económico y que, por su mayor nivel educativo, suelen ser más rápidos en aceptar diversas innovaciones. En estos grupos, la confianza en los propios derechos y el acceso a un asesoramiento jurídico de calidad son mayores, por lo que les resulta más fácil concurrir a una sala de negociaciones. Al mismo tiempo, su mayor familiaridad con el sistema jurídico les permite anticipar las consecuencias de un tratamiento adversarial de la disputa.

En las jurisdicciones en las que la ley consagra la voluntariedad como la opción dominante, cabe esperar que el flujo de causas derivadas a la mediación permanezca - por lo menos en las etapas iniciales - en un nivel bajo. Es probable también que entre los casos mediados se encuentren especialmente asuntos de monto indeterminado, así como procesos donde los montos en juego son menores. Cuando las sumas en disputa son mayores, es más frecuente que las partes prefieran esperar una decisión en los tribunales tradicionales.

La opción por un régimen voluntario de mediación supone, pues, establecer un sistema más eficaz en la resolución de los conflictos que efectivamente atiende, aunque de menor incidencia sobre los niveles generales de litigación.

En cambio, en las jurisdicciones donde el legislador prefirió la obligatoriedad de la instancia, el flu- jo de causas derivadas al sistema de mediación se vuelve masivo. El compromiso de las partes con la búsqueda de soluciones se reduce y la proporción de arreglos logrados es inferior. El sistema alternativo de resolución de conflictos resulta menos eficaz, pero su impacto sobre los volúmenes globales de litigios atendidos por la administración de justicia es mayor.

Si bien la opción por la obligatoriedad reduce un poco los niveles de satisfacción de los usuarios, las diferencias no son significativas y éstos continúan siendo altos. La mayoría de quienes han sido derivados obligatoriamente a un proceso de mediación califican positivamente las diferentes dimensiones de esa experiencia y están dispuestos a volver a usar el sistema, así como a recomendar su uso a otras personas. En particular, es importante observar que en este grupo se reconoce carácter equitativo al proceso de la mediación, y se opina mayoritariamente a favor de la obligatoriedad de la instancia.

En consecuencia, puede decirse que la elección del carácter obligatorio o voluntario de la mediación tiene consecuencias significativas sobre el funcionamiento de este modo de procesamiento de conflictos, pero no afecta el grado de legitimidad que los usuarios le reconocen. Esta conclusión resulta alentadora, habida cuenta de las divergencias en las demandas que diversos actores sociales tienen respecto de esta cuestión.

Este recorrido por los datos ha permitido señalar distintas consecuencias que la elección de uno u otro carácter de la instancia tiene sobre su funcionamiento concreto. Antes de cerrar esta discusión, permítasenos agregar que, desde nuestra perspectiva, la estrategia de cambio cultural a mediano plazo implicada en la opción por la voluntariedad resulta más adecuada para una sociedad plenamente democrática, debiendo por ello ser preferida. 


\begin{tabular}{|c|c|c|}
\hline Consecuencias sobre & $\begin{array}{c}\text { Sistema } \\
\text { Voluntario }\end{array}$ & $\begin{array}{c}\text { Sistema } \\
\text { obligatorio }\end{array}$ \\
\hline \multicolumn{3}{|l|}{ Las causas } \\
\hline Duración & Mayor & Menor \\
\hline Resultados & $\begin{array}{c}\text { Mayor proporción de } \\
\text { arreglos }\end{array}$ & $\begin{array}{c}\text { Menor proporción de } \\
\text { arreglos }\end{array}$ \\
\hline Tipo de casos & $\begin{array}{c}\text { Mayor número de causas } \\
\text { de monto indeterminado } \\
\text { o bajo }\end{array}$ & $\begin{array}{l}\text { Seleccionadas por el } \\
\text { legislador }\end{array}$ \\
\hline Los usuarios del sistema & \begin{tabular}{|c} 
Mayor proporción de \\
mujeres \\
Mayor proporción de \\
usuarios de niveles \\
sociales y educativos altos \\
Satisfacción con el \\
procedimiento algo mayor \\
Legitimidad sin variantes
\end{tabular} & $\begin{array}{l}\text { Composición más similar } \\
\text { a la población general } \\
\text { Satisfacción con el } \\
\text { procedimiento algo } \\
\text { menor } \\
\text { Legitimidad sin variantes }\end{array}$ \\
\hline \multicolumn{3}{|l|}{ La administración de justicia } \\
\hline $\begin{array}{l}\text { Flujo de casos a mediación } \\
\text { Nivel general de litigiosidad }\end{array}$ & $\begin{array}{l}\text { Inicialmente bajo } \\
\text { Poca incidencia }\end{array}$ & $\begin{array}{c}\text { Masivo } \\
\text { Efecto reductor }\end{array}$ \\
\hline
\end{tabular}

\section{Bibliografía}

- $\quad$ BARMAT, N. D. y RIVERO, S. (2001). Ley Provincial de Mediación $N^{\circ} 8858$ y su reglamentación -Comentada, Anotada, Concordada, Córdoba: Marcos Lerner, Editora Córdoba.

- $\quad$ BERGOGLIO, M. I. (1997) "Acceso a la Justicia civil: diferencias de clase": En: Anuario del Centro de Investigaciones Jurídicas y Sociales, Vol. III, Universidad Nacional de Córdoba, Córdoba, pp. 93-107.
- $\quad$ BERGOGLIO, M. I. (1999). “Desigualdades en el acceso a la justicia civil: diferencias de género". En: Anuario IV del Centro de Investigaciones Jurídicas y Sociales, UNC, Córdoba, p. 129-147.

- $\quad$ BERGOGLIO, M. I. (2003a). “La dimensión temporal durante la mediación". En: Anuario del Centro de Investigaciones Jurídicas y Sociales, Vol. VI, UNC, Córdoba. pp. 357-374. 
- $\quad$ BERGOGLIO, M. I. (2003b). La matriz del orden social: La cultura en la sociedad. Córdoba: Ciencia, Derecho y Sociedad, Facultad de Derecho y Ciencias Sociales, Universidad Nacional de Córdoba.

- $\quad$ BERGOGLIO, M. I. et al. (2001). Litigar en Córdoba. Investigaciones sociológicas sobre la litigación. Córdoba: Triunfar.

- $\quad$ BERGOGLIO, M. I., VILANOVA, J. L. (2004). La contribución de la mediación a la accesibilidad de la justicia: la visión de los actores. En: Anuario del Centro de Investigaciones Jurídicas y Sociales, Vol. VII, UNC, Córdoba.

- $\quad$ BERKOVITZ, D.; PISTOR, K. y RICHARD, J. F. (2003). "The trasplant effect". En: American Journal of Comparative Law, Vol. 51, p. 163.

- $\quad$ ENGLE, Merry S. (1990) Getting Justice and Getting even: Legal consciousness among working- class Americans, Chicago and London: The University of Chicago Press.

GARAVANO, G. (2001). “Los usuarios del sistema de justicia en Argentina". En: Nuevos enfoques para atender la demanda de justicia. México: Conferencia Regional del Banco Mundial para América Latina y el Caribe. CIDE. Mayo.

- KOURILSKY - AUGENEVEN, C. (1997) Socialisation juridique et conscience du droit, Paris: Maison des Sciences de I 'Homme.

- $\quad$ LÓPEZ GONZÁLEZ, G.; CHOLAKIAN, Claudio; et al (2002). Dossier Resolución Alternativa de Conflictos en América, Sistemas Judiciales, Año $1 \mathrm{~N}^{\circ} 2$, p. 88-104.

MENKEL MEADOW, C. (2000). Mediation: Theory, Policy and practice, Ashgate. 